\section{Neurofibromatosis type 2 a case study}

\section{H G Kritzinger MB ChB}

Department of Diagnostic Radiology University of the Free State, Bloemfontein

\section{Case presentation}

A 56-year-old black male patient presented with bilateral neuronal deafness as well as a large lump on his right forehead. Subcutaneous nodules (5 in total and not very prominent) and axillary freckles were also visible on his skin.

Computed tomography (CT) and magnetic resonance imaging (MRI) revealed intracranial masses against the skull roof (Figs 1,2), bilateral masses at the internal acoustic channels (Figs 3,4 ) as well as a mass anterior to the right temporal lobe (Fig. 5). Splaying of the internal acoustic channels was present suggesting acoustic neuromas (Fig. 3). Prominent enhancement was visible after intravenous contrast. The lesions at the skull roof showed much more enhancement than the lesions at the skull base (cerebello-pontine angle). Calcifications were also visible in the superior lesions. Biopsy of the lesion on the patient's forehead confirmed this lesion to be a meningioma. A diagnosis of neurofibromatosis type 2 (NF-2) was made. The patient did not receive surgery due to the advanced stage of the disease and was irradiated palliatively. He is currently being treated at oncotherapy. What made this case interesting was the late age at which this patient presented and that he was the first black patient to present with NF-2 at our hospital complex.

\section{Discussion}

\section{Disease}

The first probable case of NF-2 was reported in 1820 when a doctor named Wishart described a patient with multiple tumours he had examined at postmortem. Neurofibromatosis type 1 (NF-1) and NF-2

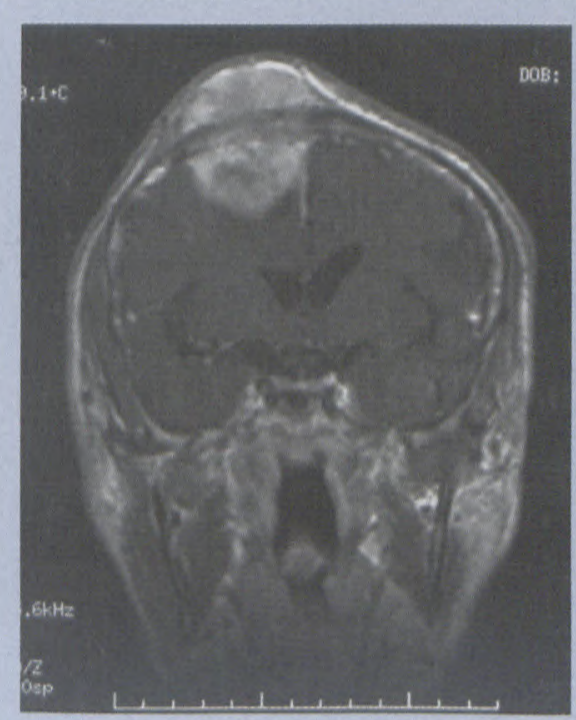

Fig. 1. Coronal T1-weighted image showing protruding mass on forehead to be of intracranial origin. are autosomal-dominant genetic disorders in which affected individuals develop both benign and malignant tumours at an increased frequency. NF-2 is a different disease from von Recklinghausen's neurofibromatosis, NF-1. NF-2 is a rare multisystem genetic disorder associated with bilateral vestibular schwannomas, spinal cord schwannomas, meningiomas, gliomas, and juvenile cataracts with a paucity of cutaneous features. All

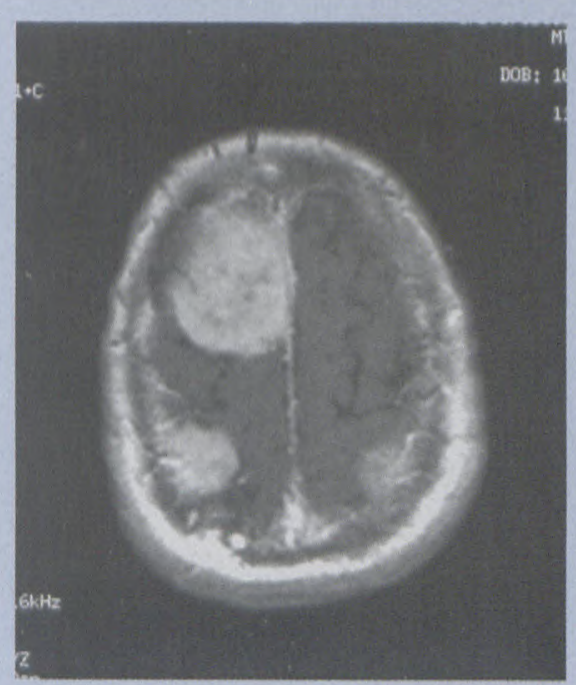

Fig. 2. Axial T1-weighted image with contrast showing meningiomas.

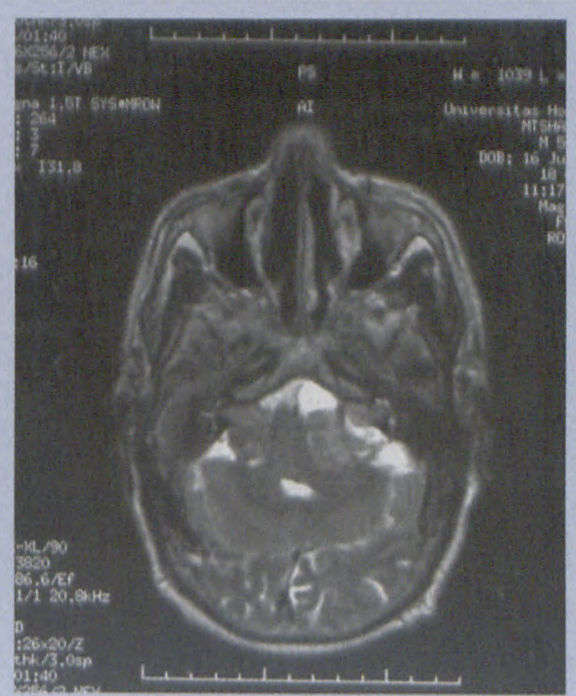

Fig. 3. Axial T2-weighted image showing vestibular schwannomas; note splaying of internal auditory meati on both sides. 


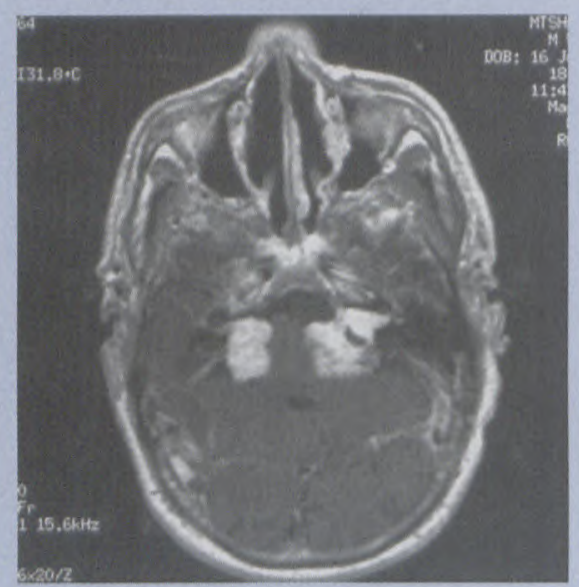

Fig. 4. Axial T1-weighted image with contrast showing vestibular schwannomas.

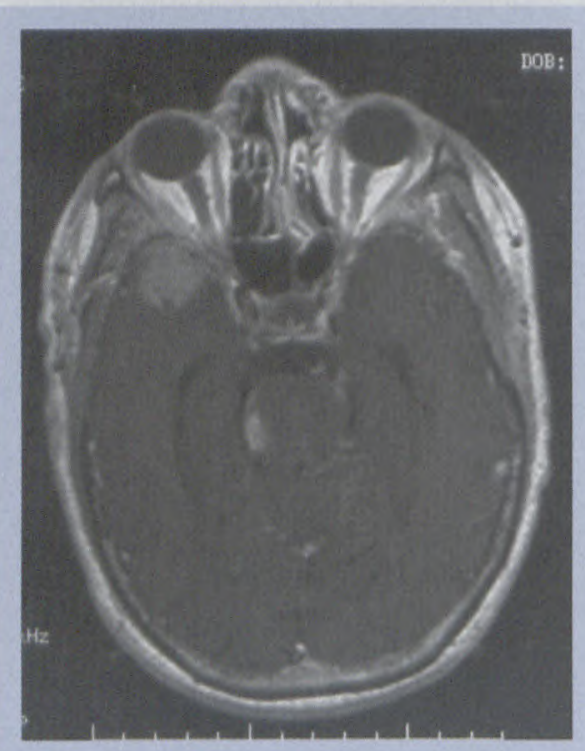

Fig. 5. Axial T1-weighted image with contrast showing mass anterior to right temporal lobe.

races and ethnic groups are affected equally with NF-2.' Although the genetic change causing NF-2 is present at conception, the clinical manifestations occur over many years. The typical age of onset of symptoms is in the late teens to early 20 s, but the age range covers the entire life span. Patients present before the age of 55 years. Some evidence indicates that age of onset of clinical symptoms is lower in maternally transmitted NF-2. While NF-2 is quite variable in severity from person to person, family stud- ies have shown some intrafamilial consistency in age of onset. ${ }^{2,3}$ Diagnosis in children is often difficult because of the absence of central nervous system (CNS) involvement at young age. ${ }^{1,4}$ The severity of the disease ranges from appearance in childhood with large numbers of crippling and fatal tumours, to mild cases with slow-growing and fewer tumours appearing later in life. This seems to be related to the difference in the NF2 gene. Data suggest that there are two types of NF-2 genes, one with later onset and bilateral vestibular schwannomas as the only usual feature and the other with earlier onset and multiple other tumours. ${ }^{5,6}$ Vestibular schwannomas are the most common and well-recognised feature of NF-2 leading to significant morbidity. Although unilateral hearing loss is the number one presenting symptom, eventually bilateral deafness would be expected in most affected individuals. Untreated vestibular schwannomas can extend locally and may result in brainstem compression, hydrocephalus and occasionally facial nerve palsy.

Dumbbell-shaped spinal cord schwannomas are quite common in NF-2 and result in significant morbidity; they present a great therapeutic challenge. Spinal cord ependymomas, astrocytomas, and meningiomas also occur, but less frequently. Intracranial meningiomas, on the other hand, are a frequent finding and may cause a variety of symptoms and CNS deficits. ${ }^{1}$

Posterior subcapsular or so-called 'juvenile cataracts' can predate CNS symptomatology. These cataracts may progress over time, leading to decreased visual acuity. A fair percentage of affected individuals are found to have retinal hamartomas or epiretinal membranes that may or may not be visually significant. ${ }^{1}$ The most common ocular abnormalities are posterior subcapsular or capsular, cortical or mixed lens opacities.

The diagnosis of NF2 in the paediatric age group requires a high degree of suspicion and should be considered in children with multiple CNS or skin tumours without cafe-au-lait spots or Lisch nodules. ${ }^{8}$

Presenting symptoms include hearing loss, ringing in the ears, and balance problems associated with vestibular nerve lesions. Individuals at risk for NF-2 should be screened carefully for early signs of hearing loss, motor or sensory changes, and visual deficits. Deafness is sometimes accompanied by tinnitus. ${ }^{6}$ Rare clinical findings are muscle weakness or wasting, seizures, vertigo, numbness and tingling. ${ }^{3}$

Differentiating clinically between the relatively common NF-1 and the rare NF-2 is occasionally problematic. Patients with NF-2 almost never have a large number of cafe-au-lait spots (although rarely 6 or more may be seen), whereas cafe-au-lait spots are numerous and ubiquitous in NF-1. Neither axillary nor inguinal freckles are common occurrences in NF-2.

Malignant transformation of benign growths is almost unheard of in NF-2, unlike NF-1. However, individuals with either NF-1 or NF-2 can develop multiple subcutaneous lesions that may be clinically indistinguishable. In NF-2 these lesions would most often be defined histologically as schwannomas or neurilemomas, while in NF-1 these would be defined histologically as neurofibromas. Subcutaneous neurofibromas are occasional findings in NF-2. A mix- 
ture between the two occurs in very rare cases.' The growth of tumours in NF-2 is unpredictable and growth rates are extremely variable. ${ }^{5,7,10}$ Most are slow-growing and can cause minimal problems for years, while others may lead to increasing problems over a few weeks.

\section{Genetics}

NF-2 is the result of an abnormal gene on chromosome 22 at Q12 (NF2), with a resulting defect in the production of the tumour-suppressor protein schwannomin, also called Merlin. Like other tumour-suppressor genes (such as p53 and $\mathrm{Rb}$ ), the normal function of NF2 is to act as a brake on cell growth and division, ensuring that cells do not divide uncontrollably, as they do in tumours. The exact molecular function of NF2 in the cell is still unknown. Schwannomin is a 595 amino acid protein. With only single amino acid abnormality, the disease is mild, whereas frame shift deletions or insertion and nonsense mutations causing truncation of schwannomin cause more severe disease. ${ }^{5,7,10} \mathrm{NF}-2$ is an autosomal-dominant genetic trait, meaning it affects both genders equally and that each child of an affected parent has a $50 \%$ chance of inheriting the gene. Half of affected individuals have NF-2 as a result of a new (de novo) gene mutation.

The estimated incidence of NF- 2 is 1 in 37000 per year, with about onethird of affected individuals representing first cases in the family as a result of new dominant mutations. ${ }^{11}$ Approximately 1000 patients in the entire United States are affected. ${ }^{5}$ There are no statistic data available for South Africa.

\section{Diagnosis}

Plain films of the spine may be helpful in evaluating scoliosis but are of limited value in looking for spinal cord tumours that may occur in NF-2. In general, MRI is the preferred technique for monitoring individuals with NF-2 who have symptoms of spinal cord lesions. ${ }^{1}$

MRI remains the mainstay for diagnosis and screening of CNS and spinal cord tumours. At-risk individuals may be monitored for CNS tumours beginning in their teens, with annual MRIs of the head done through their late 50s. MRI of the spine is indicated diagnostically when an individual presents with motor or sensory changes suggestive of a spinal cord lesion or lesions. The key point here is early detection, which may result in prompt action and provide a better outcome. However, routine MRI imaging of the spinal cord is probably not indicated for asymptomatic affected or at-risk individuals. ${ }^{12,13}$

\section{MRI findings for NF-2 are:}

1. Bilateral acoustic schwannomas. Site: superior/inferior division of vestibular nerve usually asymmetric.

2. Schwannomas of other cranial nerves. Excluding nerves without schwann cells.

3. Multiple meningiomas; intraventicular, parasagittal, sphenoid ridge, olfactory groove, along intracranial nerves.

4. Meningiomatosis = dura studded with innumerable small meningiomas.

5. Glioma of ependymal deriviation. $^{14}$

6. Spinal cord lesions: extamedullary - multiple paraspinal nerofibromas, meningiomas, schwan- nomas, syrinx associated with tumour; and (ii) intramedullary spinal cord ependymomas. ${ }^{13}$

The presence of multiple and different pathologic types of spinal tumours is highly suggestive of NF-2. ${ }^{13}$

Clear molecular diagnosis may help to modify risks for family members and prevent unnecessary testing for asymptomatic individuals who are found not to carry a gene mutation..$^{15}$

The diagnosis of NF-2 is still based largely on clinical criteria.

The updated $\mathrm{NIH}$ Consensus Development Conference criteria for confirmed or definite NF-2 differ from the original criteria only slightly and include individuals with:

1. Bilateral vestibular schwannomas visualised by MR imaging, or

2. A parent, sibling, or child with NF-2, plus (i) unilateral vestibular schwannoma detected before the age of 30 years, or (ii) any 2 of the following: meningioma, glioma, schwanno$\mathrm{ma}$, or juvenile posterior subcapsular lenticular opacity. ${ }^{6,16}$

In practice, these clinical criteria may be too rigid and may exclude individuals who should be evaluated for NF-2. The evaluation for NF-2 should never represent a single point in time, but should include long-term follow-up. If no further tumours develop during a 5 - 10-year period or if NF-2 molecular testing becomes more reliable for exclusion, then screening for possible NF-2 can be relaxed. It is recommended that a distinction be made between a 'confirmed' and a 'presumptive' diagnosis of NF-2. Individuals with multiple NF-2 manifestations and no bilateral vestibular schwannomas classified as presumptive NF-2 may represent developing NF-2, in which other 
NF-2 features have not yet manifested, or NF-2 mosaicism. It is often difficult to distinguish between NF-2 mosaicism and 'definite' NF-2.'

Other groups of individuals to be considered at risk for NF-2 and examined further include those with a family history of NF-2, persons younger than 30 years who have a unilateral vestibular schwannoma or meningioma, and those with multiple spinal tumours. In addition, individuals who have the following clinical features should be evaluated for NF-2:

1. A unilateral vestibular schwannoma plus any one of the following: meningioma, glioma, schwannoma, or juvenile posterior subcapsular lenticular opacity.

2. Multiple meningiomas plus a unilateral vestibular schwannoma.

3. Multiple meningiomas plus 1 or more of the following: glioma, schwannoma, or juvenile posterior subcapsular lenticular opacity. A highquality MRI should be performed to rule out bilateral vestibular schwannomas definitively. ${ }^{4}$

Peripheral tumours in NF-2 are usually schwannomas and not neurofibromas. Both schwannomas and neurofibromas may be useful diagnostic adjuncts in the paediatric and adolescent age groups, when severe NF-2 phenotypes typically manifest. ${ }^{4}$

Cerebral calcifications have sometimes been suggested as a diagnostic feature of NF-2. There are no convincing data to justify their inclusion, as they represent relatively nonspecific findings. In addition, CT scans that routinely detect calcification are now not the investigation of choice in NF-2. ${ }^{4}$

Now that the gene for NF-2 has been identified, analysis for diseasecausing mutations can be offered in some clinical settings. Detection rates for molecular-based testing are approximately $65 \%$; therefore, such testing is of limited use in making the diagnosis of NF-2. However, in a patient with suspected NF-2 who is still young, has a negative family history, and may eventually develop additional criteria, the identification of a specific mutation may be helpful. ${ }^{17-19}$

Predictive diagnosis by linkage analysis using markers flanking the NF2 gene is now possible in the vast majority of families with 2 or more living affected individuals. ${ }^{2}$

Once a mutation has been identified in an affected individual, a 100\% specific test is then available for that family. However, mutation detection is time-consuming and expensive and may not reveal the causative mutation. ${ }^{17-19}$ Currently these tests are not available in South Africa.

\section{References}

1. Pletcher BA, Griesemer D, Talavera F, Mack KJ, Baker MJ, Lorenzo N. Neurofibromatosis, type 2. eMedicine Journal 2002, 3: number 2. www.eMedicine.com/Neuro/

2. Kanter WR, Eldridge R, Fabricant R, Allen JC, Koerber T. Central neurofibromatosis with bilateral acoustic neuroma: genetic, clinical and biochemical distinctions from peripheral neurofibromatosis. Neurology 1980; 30: 851-859.

3. Evans DG, Huson SM, Donnai D, et al. A clinical study of type 2 neurofibromatosis. QJM 1992; 84: 603-618.

4. Gutmann DH, Aylsworth A, Carey JC, et al. The diagnostic evaluation and multidisciplinary management of neurofibromatosis 1 and neu- rofibromatosis 2. JAMA 1997; 278: 51-57.

5. Malis LI. Nuances in acoustic neuroma surgery. Neurosurgery 2001; 49: 337-341.

6. Evans DG, Huson SM, Donnai D, et al. A genetic study of type 2 neurofibromatosis in the United Kingdom. I, Prevalence, mutation rate, fitness, and confirmation of maternal transmission effect on severity. J Med Genet 1992; 29: 841-846. Comment in: J Med Genet 2001; 38: 783-784.

7. Ruttledge $\mathrm{MH}$, Andermann AA, Phelan $\mathrm{CM}$, et al. Type of mutation in the neurofibromatosis type 2 gene (NF2) frequently determines severity of disease. Am J Hum Genet 1996; 59: 331 342.

8. Mautner VF, Tatagiba M, Guthoff R, Samii M, Pulst SM. Neurofibromatosis 2 in the pediatric age group. Neurosurgery 1993; 33(1): 92-96.

9. Mautner VF, Lindenau M, Baser ME, Kluwe L, Gottschalk J. Skin abnormalities in neurofibromatosis 2. Arch Dermatol 1997; 133: 1539-1543.

10. Parry DM, MacCollin MM, Kaiser-Kupfer MI et al. Germ-line mutations in the neurofibromatosis 2 gene: correlations with disease verity and retinal abnormalities. Am J Hum Genet 1996; 59: 529-539.

11. Merel P, Hoang-Xuan K, Sanson M, et al. Screening for germ-line mutations in the NF2 gene. Genes Chromosomes Cancer 1995; 12: 117 127.

12. McCormick PC, Torres R, Post KD, Stein BM. Intramedullary ependymoma of the spinal cord. J Neurosurg 1990; 72: 523-532.

13. Mautner VF, Tatagiba M, Lindenau M, et al Spinal tumours in patients with neurofibromatosis type 2: MR imaging study of frequen$\mathrm{cy}$, multiplicity, and variety. Am J Roentgenol 1995; 165: 951-955. Erratum in: AJR Am J Roentgenol 1996; 166: 1231.

14. Danhert W. Radiological Review Manual. 4th ed. Philadelphia: Williams and Wilkins, 1999: 261.

15. Sainio M, Strachan T, Blomstedt G, et al. Presymptomatic DNA and MRI diagnosis of neurofibromatosis 2 with mild clinical course in an extended pedigree. Neurology 1995; 45: 1314-1322.

16. Evans DGR, Huson SM, Donnai D, et al. A clinical study of type 2 neurofibromatosis. QJM 1992; 304: 603-618.

17. Evans DGR, Bourn D, Wallace A, Ramsden RT, Mitchell JD, Strachan T. Diagnostic issues in a family with late onset type 2 neurofibromatosis. JMed Genet 1995; 32: 470-474.

18. Bourn D, Carter SA, Mason S, Gareth D, Evans $\mathrm{R}$, Strachan T. Germline mutations in the neurofibromatosis type 2 tumour suppressor gene. Hum Mol Genet 1994; 3: 813-816.

19. Merel P, Hoang-Xuan K, Sanson M, et al. Screening for germ-line mutations in the NF2 gene. Genes Chromosomes Cancer 1995; 12: 117 127. 